\title{
Disinfecting dystonia? Drug discovery using worms identifies an antibiotic as a neuroprotective lead molecule for movement disorders
}
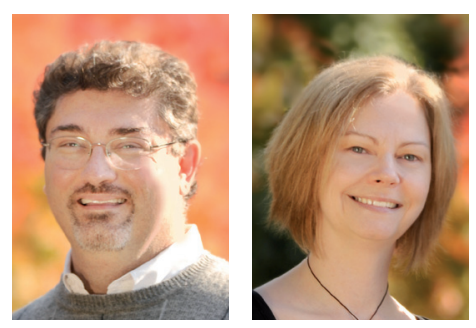

"...the prospect that torsinA-modifying drugs may serve as neuroprotective agents represents a novel therapeutic strategy for not only dystonia, but also Parkinson's disease."

Guy A Caldwell ${ }^{\dagger}$ \& Kim A Caldwell

Department of Biological Sciences, The University of Alabama, Box 870344, Tuscaloosa, AL, 35487, USA †Author for correspondence: Tel.: +1 2053489926 = Fax: +1 2053481786 = gcaldwel@bama.ua.edu

The combination of microscopic worms and an antibiotic may seem an unlikely pairing in the quest to uncover new therapeutic leads to combat neurological diseases. Nevertheless, it doesn't take an extended interaction with patients suffering from human movement disorders to recognize that the time for conventional thinking has long since passed. Movement disorders represent a substantial societal burden afflicting millions of individuals worldwide. Current medicines used for these diseases are largely limited to management of symptoms and do not address the root causes of disease. Experimental strategies that exploit the various strengths of cellular and animal model systems serve to accelerate the discovery and translational process. In this regard, using a combination of worms, mice and human patient cells, our laboratory and collaborators have recently reported that the common antibiotic, ampicillin, represents a putative lead molecule for drug development aimed at the treatment of dystonia and, potentially, Parkinson's disease [1].

Early-onset generalized or torsion dystonia is the most common and severe heritable form of 'dystonia', a clinical term inclusive of related, yet genetically or symptomatically distinct conditions. Dystonia patients characteristically suffer sustained and involuntary muscle contractions that frequently cause repetitive movements or abnormal and often painful postures [2]. Despite mechanistic advances in comprehending dystonias, these remain poorly understood dysfunctions of the CNS. The seminal discovery of a single in-frame codon deletion in the human DYT1 gene as being linked to early-onset generalized dystonia, by the lab of Xandra Breakefield at Harvard University in 1997, provided the first molecular clue to the underlying cause of this disorder - the protein product of DYT1, termed torsinA [3].

'...as a prerequisite to effective drug development, ourselves and others set out to establish biological assays wherein quantitative changes in torsinA activity could be readily discerned in response to small molecule modifiers."

Early-onset generalized (DYT1) dystonia exhibits limited pathology and is a nondegenerative neurological disorder, thereby rendering it a good candidate for drug therapy. In the 13 years since the discovery of torsin A, the vast majority of research on this protein has focused on aspects of its tissue expression, intracellular localization of the mutant form, and putative protein interactors [4-6]. While these collective studies have substantially enlightened our molecular understanding of the cellular consequences of the torsin A mutation, limited insights in to torsin A function have been reported. Therefore, as a prerequisite to effective drug development, ourselves and others set out to establish biological assays wherein quantitative changes in torsin A activity could be readily discerned in response to small molecule modifiers.
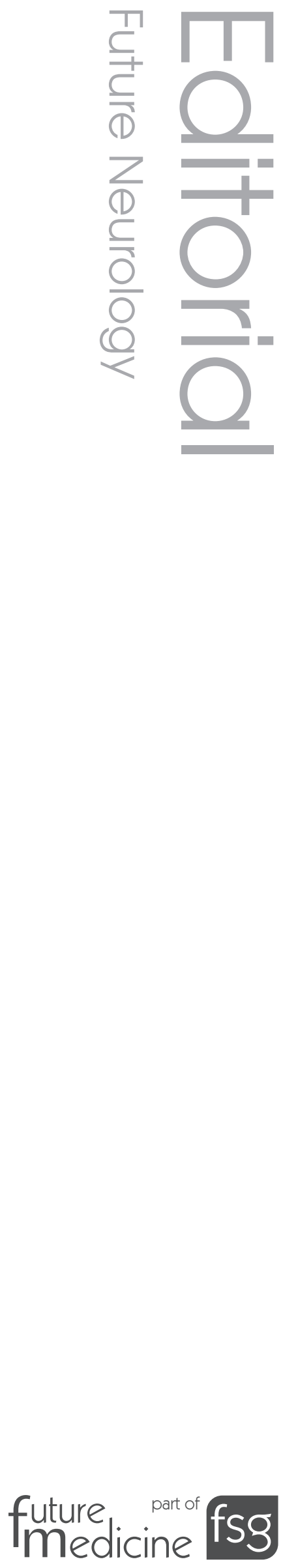
Torsin A is a member of the AAA+ family of cellular ATPases, a diverse collection of proteins that includes multiple molecular chaperones [7]. In 2003, our laboratory explored the hypothesis that torsin A functioned as a protein chaperone through generating transgenic strains of the nematode Caenorhabditis elegans co-expressing variants of human torsin A [8]. These animals were engineered to also co-express polyglutamine-repeat fusions to the green fluorescent protein (GFP) that enable visualization of protein misfolding in vivo. Co-expression of normal, but neither mutant torsin A nor a combination of mutant and normal torsin A, significantly suppressed polyglutamine-expansion dependent protein aggregation. At the time, this work represented evidence of torsin A chaperone function, that has also recently been recapitulated in vitro [9], but it equally established an excellent assay for quantitative evaluation of effectors of torsin A activity.

"It is often the case that investigation of more rare diseases leads to findings that may impact more common ones."

At the urging of proactive dystonia patients and medical foundations, we conducted a small molecule screen of a library of 240 chemically diverse off-patent drugs that had been prescreened for toxicity to $C$. elegans. Our strongest consideration in selecting this set of compounds was the hope of an accelerated clinical trial, with reduced expense, for a repurposed molecule, as opposed to the lengthy clinical approval process associated with a new chemical entity. Through multiple rounds of screening and rescreening in the aforementioned distinct transgenic $C$. elegans strains, two classes of molecules were revealed as having a capacity to specifically enhance normal (or 'wild-type') torsinA activity in suppression of protein misfolding. These molecules, which did not exhibit an effect on mutant torsin A animals or the absence of torsin $\mathrm{A}$, included two different quinolones and a $\beta$-lactam antibiotic. An analysis of cousin molecules in both classes revealed that many of these molecules were active as well. A structure-activity relationship analysis was performed using all positive and negative compounds from the screen. Notably, while the $\beta$-lactam antibiotics and quinolones have completely different mechanisms of action, a pharmacophoric model revealed an overlapping region of commonality that was separate from the antibiotic core regions of these molecules [1]. Thus, it is possible that the antibiotics simply provide a structural scaffold necessary for the shared protective torsinA-dependent activity observed in vivo.

While C. elegans is both cost effective and efficient for purposes of drug discovery, it is certainly essential to validate molecular leads in mammalian systems. Therefore, in collaboration with the Breakefield lab, we next examined the potential for the torsinA-modifying drugs identified through our worm screens to impact a readout associated with fibroblast cultures taken directly from dystonia patients. Fibroblasts from affected DYT1 patients exhibit a deficit in protein processing through the endoplasmic reticulum, the primary site of normal torsin A localization, measured by levels of Gaussia luciferase (Gluc), a fluorescent reporter [10,11]. These studies showed that endogenous torsinA activity was restored to normal levels seen in control fibroblasts by treatment with the $\beta$-lactam antibiotic, ampicillin, but not the quinilone, enoxcin.

Successful translation of these findings to the clinic is contingent upon evidence of efficacy in rodent models of disease. The laboratory of Yuqing Li (University of Alabama at Birmingham, USA) developed a genetic mimic of DYT1 dystonia in mice via knock-in of the mutant torsinA allele [12] . Heterozygous mice exhibit abnormal locomotive behavior whereby they experience increased slips in a beam walking assay. Strikingly, this aberrant movement was rescued following intraperitoneal administration of ampicillin at dosages akin to those commonly used for its action as an antibiotic. Furthermore, striatial protein extracts taken from treated normal versus DYT1 mice indicated that ampicillin served to stabilize torsinA levels in the mutant background, thereby providing preliminary insight into one potential mechanism of action for this drug.

\section{“...continued investigation of the normal role that torsinA plays in mediating intracellular dynamics will likely reveal additional aspects of its functionality that can be further exploited to enhance neuroprotective strategies."}

It is often the case that investigation of more rare diseases leads to findings that may impact more common ones. In this context, it is significant to note that our laboratory previously reported that torsin A protects dopamine (DA) neurons of C. elegans from age-dependent neurodegeneneration associated with overproduction of human $\alpha$-synuclein [13], an established 
aspect of the most prevalent movement disorder, Parkinson's disease. These data correlated with studies that showed torsin A suppresses $\alpha$-synuclein aggregation both in $C$. elegans and mammalian cell cultures [14,15]. As part of our drug screening efforts, we incorporated this nematode DA neurodegeneration model as yet another in vivo test of our small molecule effectors of torsin A activity. Most significantly, ampicillin also enhanced neuroprotection against $\alpha$-synuclein toxicity within $C$. elegans DA neurons in a torsinA-dependent manner. Although the DYT1 gene is widely expressed in most human organs, Augood et al. demonstrated that it is significantly enriched in basal ganglia nuclei, including the DA neurons of the substantia nigra [16]. Therefore, the prospect that torsinA-modifying drugs may serve as neuroprotective agents represents a novel therapeutic strategy for not only dystonia, but also Parkinson's disease.

\section{"The therapeutic pipeline for human movement disorders benefits from coordinated drug discovery efforts that run the spectrum of animal models and innovative assays available in the scientific community."}

The therapeutic pipeline for human movement disorders benefits from coordinated drug discovery efforts that run the spectrum of animal models and innovative assays available in the scientific community. Exploiting the variety of strengths of distinct systems can serve to accelerate the path to the clinic and minimize costs in the process. Our strategy focused on the utility of combining functional assays in an invertebrate model system with downstream validation in human patient cells and a transgenic mouse model. Our goal was the identification of a lead compound that activates torsinA, a previously unexploited molecular target for movement disorders. Unfortunately, as an antibiotic, ampicillin is simply not an ideal treatment, as these diseases require chronic administration paradigms. Experienced clinicians may potentially elect to explore alternate dosing strategies that attempt to bypass the precarious nature of persistent antibiotic use, but, in general, the long-term use of antibiotics is highly undesirable due to the potential for antibiotic-resistant microorganisms and dangers to essential bacterial flora of humans. In this regard, medicinal chemistry efforts to discern and clarify bioactive compounds that retain the capacity to activate torsinA-mediated protection yet lack antimicrobial activity are underway in our laboratory to circumvent these drawbacks. In the interim, continued investigation of the normal role that torsinA plays in mediating intracellular dynamics will likely reveal additional aspects of its functionality that can be further exploited to enhance neuroprotective strategies. In the end, a hopeful outcome from our efforts can be surmised: as the worm leads, mouse and man shall follow.

\section{Acknowledgements}

We wish to thank our collaborators in the Breakefield and Li laboratories for their contributions and insights. Special thanks go to Songsong Cao and Amber Clark Buckley for their dedicated efforts in the discovery process, and John Holaday for his vision and support.

\section{Financial \& competing interests disclosure}

The authors serve as scientific advisors to QRxPharma, $L t d$. from whom they receive compensation and manage a sponsored research agreement for The University of Alabama.The authors have no other relevant affiliations or financial involvement with any organization or entity with a financial interest in or financial conflict with the subject matter or materials discussed in the manuscript apart from those disclosed.

No writing assistance was utilized in the production of this manuscript.

\section{Bibliography}

1. Cao S, Hewett JW, Yokoi F et al: Chemical enhancement of torsinA function in cell and animal models of torsion dystonia. Dis. Model. Mech. 3(5-6), 386-396 (2010).

2. Breakefield XO, Blood AJ, Li Y et al.: The pathophysiological basis of dystonias. Nat. Rev. Neurosci. 9(3), 222-234 (2008).
3. Ozelius LJ, Hewett JW, Page CE et al.: The early-onset torsion dystonia gene (DYTI) encodes an ATP-binding protein. Nat. Genet. 17(1), 40-48 (1997).

4. Gonzalez-Alegre P, Paulson HL: Aberrant cellular behavior of mutant torsin $\mathrm{A}$ implicates nuclear envelope dysfunction in DYT1 dystonia J. Neurosci. 24(11), 2593-2601 (2004).

5. Goodchild RE, Dauer WT: Mislocalization to the nuclear envelope: an effect of the dystonia-causing tosin A mutation. Proc. Natl. Acad. Sci. USA 101(3), 847-852 (2004).
6. Naismith TV, Heuser JE, Breakefield XO, Hanson PI: Torsin A in the nuclear envelope. Proc. Natl. Acad. Sci. USA 101(20), 7612-7617 (2004).

7. Hanson PI, Whitehouse SW: AAA+ proteins: have engine, will work. Nat. Rev. Mol. Cell. Biol. 6, 519-529 (2005).

8. Caldwell GA, Cao S, Sexton EG, Gelwix CC Bevel JP, Caldwell KA: Suppression of polyglutamine-induced protein aggregation in Caenorhabditis elegans by torsin proteins. Hum. Mol. Genet. 12(3), 307-319 (2003). 
9. Burdette AJ, Churchill PF, Caldwell GA, Caldwell KA: The early-onset torsion dystonia protein, torsin A, displays molecule chaperone activity in vitro. Cell Stress Chaperones DOI 10.1007/s12192-010-01732 (2010) (Epub ahead of print).

10. Badr CE, Hewett JW, Breakefield XO, Tannous BA: A highly sensitive assay for monitoring the secretory pathway and ER stress. PLoS One 2(6), E571 (2007).

11. Hewett JW, Tannous B, Niland BP et al.: Mutant torsinA interferes with protein processing through the secretory pathway in DYT1 dystonia cells. Proc. Natl Acad. Sci. USA 104(17), 7271-7276 (2007).
12. Dang MT, Yokoi F, McNaught KS et al.: Generation and characterization of Dyt1 $\triangle$ GAG knock-in mouse as a model for early-onset dystonia. Exp. Neurol. 196(2), 452-263 (2005).

13. Cao S, Gelwix CC, Caldwell KA, Caldwell GA: Torsin-mediated protection from cellular stress in the dopaminergic neurons of Caenorhabditis elegans. J. Neurosci. 25(15), 3801-3812.

14. McLean PJ, Kawamata H, Shariff S et al:: Torsin A and heat shock proteins act as molecular chaperones: suppression of $\alpha$-synuclein aggregation. J. Neurochem. 83(4), 846-854 (2002).
15. Hamamichi S, Rivas RN, Knight AL, Cao S, Caldwell KA, Caldwell GA: Hypothesisbased RNAi screening identifies neuroprotective genes in a Parkinson's disease model. Proc. Natl Acad. Sci. USA 105(2), 728-733.

16. Augood SJ, Martin DM, Ozelius LJ, Breakefield XO, Penney JB Jr, Standaert DG: Distribution of the mRNAs encoding torsin A and torsinB in the normal adult human brain. Ann. Neurol. 46(5), 761-769 (1999). 